\title{
New Parameter of CG-Method for Unconstrained Optimization
}

Hamsa Th. Chilmeran

hamsathrot@uomosul.edu.iq

Department of Mathematics

College of Computer Science and Mathematics
Satia A. Al-Taie

altaiesatia@uomosul.edu.iq

Computer Center

Enaam Gh. Saeed

enaamghanim@uomosul.edu.iq

Department of Mathematics

College of Computer Science and Mathematics

University of Mosul, Mosul, Iraq

\section{Received on: 02/10/2018}

Accepted on: 28/03/2019

\section{ABSTRACT}

In this paper, we derived a new parameter by equating the modified QN direction which is suggested by [7] and the standard CG method which satisfied the sufficient descent condition and the global convergence under some assumptions. The numerical experiment of the new algorithm perform better than previous standard algorithm depending on the number of calling the function (NOF) and number of iterations (NOI). Keywords: CG-method, QN-direction, global convergence.

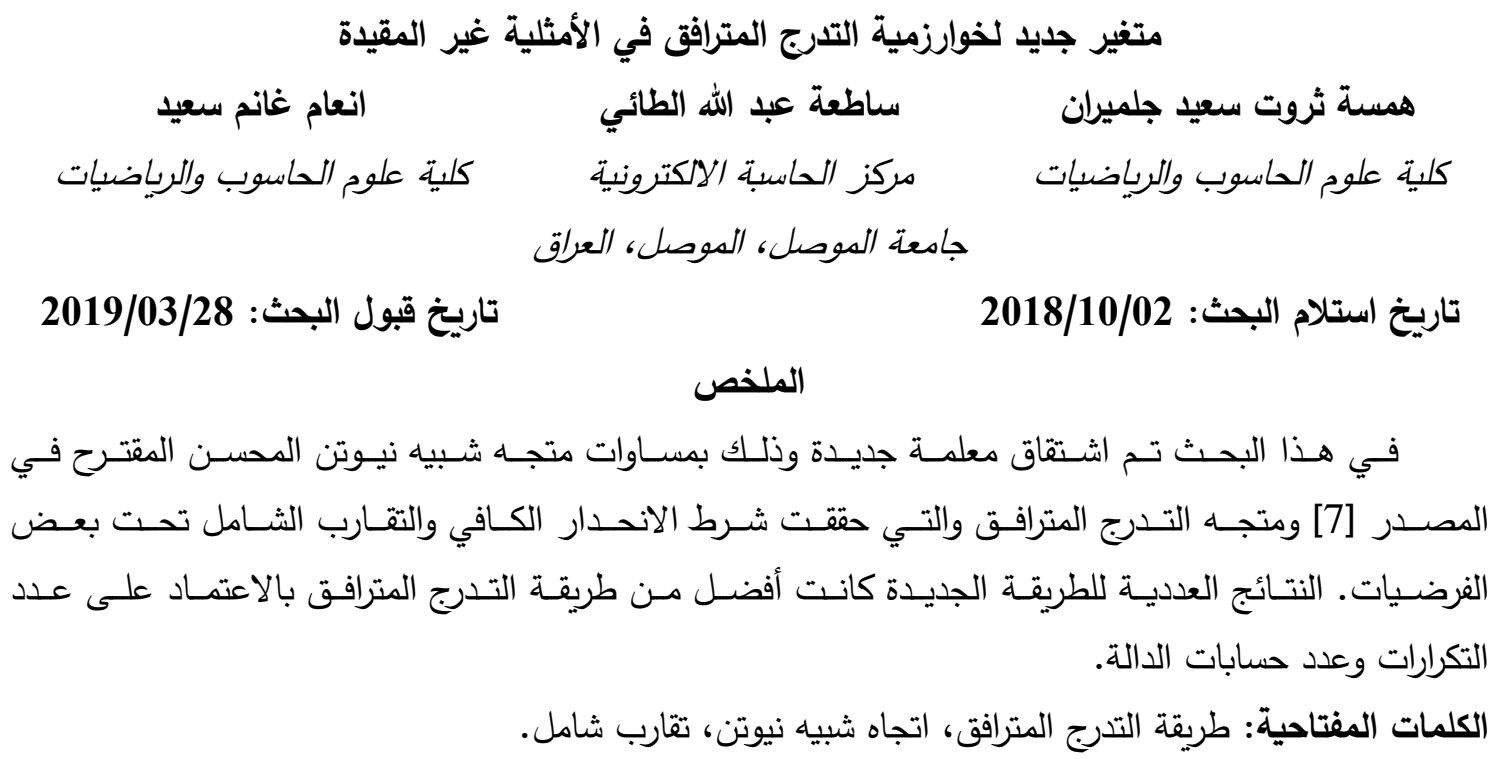

\section{Introduction}

The conjugate gradient algorithm represents an operatively idea to find minimum optimization problem with the form:

Min $f(x), x \in R^{n}$

Where $f: R^{n} \rightarrow R$ is continuously differentiable function with available gradient.

CG algorithm needs the information of first derivative, where $g(x)$ denotes the gradient and $x_{0}$ be the first approximate solution of (1). A sequence of solutions will be generated when a standard CG algorithm is used to solve (1), in which $x_{k+1}=x_{k}+\alpha_{k} d_{k}, k=0,1, \ldots$,

Such that $\alpha_{k}$ is the step length, selected to minimize $f\left(x_{k}\right)$ through $d_{k}$, its computation sometimes based on the weak Wolfe-conditions:

$$
\begin{aligned}
& f\left(x_{k}+\alpha_{k} d_{k}\right)-f\left(x_{k}\right) \leq \tilde{\delta} \alpha_{k} g_{k}^{T} d_{k} \\
& g\left(x_{k}+\alpha_{k} d_{k}\right)^{T} d_{k} \geq \sigma g_{k}^{T} d_{k}
\end{aligned}
$$

and the strong Wolfe-conditions:[8] 
) 5(

$$
\begin{gathered}
f\left(x_{k}+\alpha_{k} d_{k}\right)-f\left(x_{k}\right) \leq \tilde{\delta} \alpha_{k} g_{k}^{T} d_{k} \\
\left|g\left(x_{k}+\alpha_{k} d_{k}\right)^{T} d_{k}\right| \leq-\sigma g_{k}^{T} d_{k}
\end{gathered}
$$

Where $\tilde{\delta} \in(0,1)$ and $\sigma \in\left(\tilde{\delta}, \frac{1}{2}\right)$.

The search direction $\left(d_{k}\right)$ defined by:

$$
d_{k+1}=\left\{\begin{array}{lr}
-g_{k+1} & \text { If } k=0 \\
-g_{k+1}+\beta_{k} d_{k} & \text { If } k>0
\end{array}\right.
$$

Where $\beta_{k}$ is called conjugacy parameter, Some formulas for $\left(\beta_{k}\right)$ are given by: $\beta_{k}^{H S}=\frac{g_{k+1}^{T} y_{k}}{d_{k}^{T} y_{k}} \quad$ (Hestenes - Stiefel, (1952)), [5]

$\beta_{k}^{F R}=\frac{g_{k+1}^{T} g_{k+1}}{g_{k}^{T} g_{k}} \quad($ Fletcher - Reeves (FR), (1964)), [3]

$\beta_{k}^{C D}=\frac{g_{k+1}^{T} g_{k+1}}{-d_{k}^{T} g_{k}} \quad($ Fletcher $(\mathrm{CD}),(1987)),[4]$

$\beta_{k}^{L S}=\frac{g_{k+1}^{T} y_{k}}{-d_{k}^{T} g_{k}} \quad$ (Liu-Storey (LS), (1991)), [6]

$\beta_{k}^{D Y}=\frac{g_{k+1}^{T} g_{k+1}}{d_{k}^{T} y_{k}} \quad$ (Dai-Yun (DY) (1999)), [3]

$\beta_{k}^{N}=\frac{g_{k+1}^{T} y_{k}}{d_{k}^{T} y_{k}}-2 \frac{\left\|y_{k}\right\|^{2}}{\left(d_{k}^{T} y_{k}\right)^{2}} g_{k+1}^{T} d_{k}$ (Andrie.N.(2008)), [1]

where $y_{k}=g_{k+1}-g_{k}$.

M. Mamat and I. Mohd In [7] derived a Hybrid Modified BFGS Algorithms $B_{k+1}$ defined by

$$
d_{k+1}=-B_{k+1}^{-1} g_{k+1}-\lambda g_{k+1}
$$

Where $\lambda$ is a constant, $\lambda \in(0,1)$

$$
\begin{aligned}
& \text { And } B_{k+1}=B_{k}+\frac{y_{k}^{T} y_{k}}{y_{k}^{T} s_{k}}-\frac{B_{k} s_{k}^{T} s_{k} B_{k}^{T}}{s_{k}^{T} B_{k} s_{k}}, \\
& B_{k+1} \text { is the approximation of the Hessian } H_{i, j}=\frac{\partial f}{\partial x_{i} \partial x_{j}} \text {, satisfies } \\
& B_{k+1} s_{k}=y_{k}
\end{aligned}
$$

\section{The new parameter $\left(\beta_{k}\right)$ :}

In this paper, we derive a new parameter $\beta_{k}$ depending on the two directions, the first direction defined by (7) and the second defined by (15)

equating (7) and (15) we get:

$-g_{k+1}+\beta_{k} s_{k}=-B_{k+1}^{-1} g_{k+1}-\lambda g_{k+1}$ 
Multiplying (17) with $s_{k}^{T} B_{k+1}$

$-s_{k}^{T} B_{k+1} g_{k+1}+s_{k}^{T} B_{k+1} \beta_{k} s_{k}=-s_{k}^{T} g_{k+1}-\lambda s_{k}^{T} B_{k+1} g_{k+1}$

Substitute (16) in (18), we get

$\therefore-y_{k}^{T} g_{k+1}+y_{k}^{T} \beta_{k} s_{k}=-s_{k}^{T} g_{k+1}-\lambda y_{k}^{T} g_{k+1}$

And the new parameter $\beta_{k}$ defined as:

$\beta_{k}=\frac{-s_{k}^{T} g_{k+1}+(1-\lambda) y_{k}^{T} g_{k+1}}{s_{k}^{T} y_{k}}$

If ELS and $\lambda=0$, scalar $\beta_{k}$ reduce to the standard scalar $\beta_{k}^{H S}$

$d_{k+1}=-g_{k+1}+\frac{-s_{k}^{T} g_{k+1}+(1-\lambda) y_{k}^{T} g_{k+1}}{s_{k}^{T} y_{k}} s_{k}$

\section{The Algorithm of the New Modified Parameter $s$}

Step 1: given $x_{0} \in R^{n}$, Set $k=0$.

Step 2 : let $d_{0}=-g_{0}$

Step 3 : Find the step length $\alpha_{k}>0$, satisfying (3), (4), and set

$x_{k+1}=x_{k}+\alpha_{k} d_{k}$.

Step 4: if $\left\|g_{k}\right\| \leq 10^{-5}$, then stop

Step 5: Otherwise, compute the direction using (19)

Step 6: if $k=n$, or $\frac{\left|g_{k}^{T} g_{k+1}\right|}{\left\|g_{k+1}\right\|^{2}} \geq 0.2$ [9], then go to step 2 else set $k=k+1$, go to step3.

\section{The sufficient descent condition for the new direction}

Theorem (1) :The new direction $d_{k}$ which is given in (19) satisfies the sufficient descent condition $g_{k+1}^{T} d_{k+1}<0$ for all $k$.

For the prove, , it is necessary to assume the following basic assumption:

Assumption 1:The objective function $f$ in equation (1) is differentiable with Lipschitz continuous gradient on level set see [2]

Proof the theorem :

Multiplying (19) by $\frac{g_{k+1}}{\left\|g_{k+1}\right\|^{2}}$, we get:

$d_{k+1}^{T} g_{k+1}=-\left\|g_{k+1}\right\|^{2}+\frac{-s_{k}^{T} g_{k+1}+(1-\lambda) y_{k}^{T} g_{k+1}}{s_{k}^{T} y_{k}} \cdot \frac{s_{k}^{T} g_{k+1}}{\left\|g_{k+1}\right\|^{2}}$

$\frac{d_{k+1}^{T} g_{k+1}}{\left\|g_{k+1}\right\|^{2}}+1=\frac{-s_{k}^{T} g_{k+1}+(1-\lambda) y_{k}^{T} g_{k+1}}{s_{k}^{T} y_{k}} \cdot \frac{s_{k}^{T} g_{k+1}}{\left\|g_{k+1}\right\|^{2}}$

Since from weak Wolf condition (4) we have: 
$-s_{k}^{T} g_{k+1} \leq-\sigma d_{k}^{T} g_{k}$

Substitute (22) in (21) we have:

$\frac{d_{k+1}^{T} g_{k+1}}{\left\|g_{k+1}\right\|^{2}}+1 \leq \frac{-\sigma d_{k}^{T} g+(1-\lambda) y_{k}^{T} g_{k+1}}{s_{k}^{T} y_{k}} \cdot \frac{s_{k}^{T} g_{k+1}}{\left\|g_{k+1}\right\|^{2}}$

From the Norm definition:

$g_{k+1}^{T} y_{k} \leq\left\|g_{k+1}\right\|\left\|y_{k}\right\|$ and since :

$s_{k}^{T} g_{k+1} \leq s_{k}^{T} y_{k}$,

we have

$\frac{d_{k+1}^{T} g_{k+1}}{\left\|g_{k+1}\right\|^{2}}+1 \leq \frac{-\sigma d_{k}^{T} g_{k}+(1-\lambda)\left\|g_{k+1}\right\|\|\| y_{k} \|}{s_{k}^{T} y_{k}} \cdot \frac{s_{k}^{T} y_{k}}{\left\|g_{k+1}\right\|^{2}}$

$\frac{d_{k+1}^{T} g_{k+1}}{\left\|g_{k+1}\right\|^{2}}+1 \leq \frac{-\sigma d_{k}^{T} g_{k}+(1-\lambda)\left\|g_{k+1}\right\| .\left\|y_{k}\right\|}{\left\|g_{k+1}\right\|^{2}}$

Since $d_{k}^{T} g_{k} \leq \frac{-d_{k}^{T} y_{k}}{(\sigma+1)}$

$\therefore \frac{d_{k+1}^{T} g_{k+1}}{\left\|g_{k+1}\right\|^{2}}+1 \leq \frac{\sigma \frac{d_{k}^{T} y_{k}}{\sigma+1}+(1-\lambda)\left\|g_{k+1}\right\| .\left\|y_{k}\right\|}{\left\|g_{k+1}\right\|^{2}} \leq \frac{\sigma \frac{L\left\|s_{k}\right\|^{2}}{\sigma+1}+(1-\lambda)\left\|g_{k+1}\right\|\|\| y_{k} \|}{\left\|g_{k+1}\right\|^{2}}$

Let $C=\frac{\sigma \frac{L\left\|s_{k}\right\|^{2}}{\sigma+1}+(1-\lambda)\left\|g_{k+1}\right\| .\left\|y_{k}\right\|}{\left\|g_{k+1}\right\|^{2}}$

$\therefore d_{k+1} g_{k+1} \leq(-1+c)\left\|g_{k+1}\right\|^{2} \leq-(1-c)\left\|g_{k+1}\right\|^{2}$

$\therefore d_{k+1} g_{k+1} \leq-(1-c)\left\|g_{k+1}\right\|^{2}$

The proof is complete

\section{The Global Convergence of the New Proposed Parameter for CG-Method}

Theorem (2). Let the assumption (A) hold and the conjugate gradient methods (2) and (7) hold, $d_{k}$ is a descent direction where $\lambda_{k}$ is satisfied (5) and (6) [8]

$$
\text { if } \begin{aligned}
& \sum_{k \geq 1} \frac{1}{\left\|d_{k+1}\right\|}=\infty \\
\therefore & \liminf _{k \rightarrow \infty}\left\|g_{k}\right\|=0
\end{aligned}
$$

Theorem: let that the assumption (A) holds .consider the algorithm in (2), $d_{k}$ is defined by (19), then the new algorithm either stops at stationary point i.e $\left\|g_{k}\right\|=0$ or $\liminf _{k \rightarrow \infty}\left\|g_{k}\right\|=0$

Proof: 


$$
\begin{aligned}
& d_{k+1}=-g_{k+1}+\frac{-s_{k}^{T} g_{k+1}+(1-\lambda) y_{k}^{T} g_{k+1}}{s_{k}^{T} y_{k}} s_{k} \\
& \left\|d_{k+1}\right\|=\left|-g_{k+1}+\frac{-s_{k}^{T} g_{k+1}+(1-\lambda) y_{k}^{T} g_{k+1}}{s_{k}^{T} y_{k}} s_{k}\right| \\
& \left\|d_{k+1}\right\| \leq \| g_{k+1}||+\left|\frac{-s_{k}^{T} g_{k+1}+(1-\lambda) y_{k}^{T} g_{k+1}}{s_{k}^{T} y_{k}} s_{k}\right| \\
& \left\|d_{k+1}\right\| \leq\left\|g_{k+1}\right\|+\left|\frac{-s_{k}^{T} g_{k+1}}{s_{k}^{T} y_{k}}\right|+\left|\frac{(1-\lambda) y_{k}^{T} g_{k+1}}{s_{k}^{T} y_{k}}\right| \mid s_{k} \| \\
& \left\|d_{k+1}\right\| \leq\left\|g_{k+1}\right\|+\left|\frac{-s_{k}^{T} g_{k+1}}{s_{k}^{T} y_{k}}\right|+(1-\lambda)\left|\frac{y_{k}^{T} g_{k+1}}{s_{k}^{T} y_{k}}\right|\left\|s_{k}\right\|
\end{aligned}
$$

Since the new direction is descent and from convex condition and Lipchitz condition we have

$$
\begin{aligned}
& u\|s\|^{2} \leq s_{k}^{T} y_{k} \leq L\|s\|^{2} \\
& \left\|d_{k+1}\right\| \leq\left\|g_{k+1}\right\|+\frac{\sigma\left\|g_{k}\right\|^{2}}{u\left\|s_{k}\right\|^{2}}+(1-\lambda)\left|\frac{\left\|g_{k+1}\right\| .\left\|y_{k}\right\|}{u\left\|s_{k}\right\|^{2}}\right|\left\|s_{k}\right\|
\end{aligned}
$$

Let

$$
\tilde{\gamma}=\left\|g_{k+1}\right\|+\frac{\sigma\left\|g_{k}\right\|^{2}}{u\left\|s_{k}\right\|^{2}}+(1-\lambda)\left|\frac{\left\|g_{k+1}\right\| \cdot\left\|y_{k}\right\|}{u\left\|s_{k}\right\|^{2}}\right|\left\|s_{k}\right\| \geq 0
$$

Then

$$
\begin{aligned}
& \left\|d_{k+1}\right\| \leq \tilde{\gamma} \\
& \sum_{k \geq 1} \frac{1}{\left\|d_{k+1}\right\|} \geq \sum_{k \geq 1} \frac{1}{\gamma} \cdot \sum_{k \geq 1} 1=\infty \\
& \liminf \| \underset{k \rightarrow \infty}{g_{k} \|}=0
\end{aligned}
$$

The proof is complete

\section{The Numerical Result}

In this part we use "Visual Fortran language" to implement the new algorithm defined in (19) for presenting the computational performance of (27) unconstraint test functions in [1], All algorithms achieve the strong Wolf line search conditions and $\left\|g_{k}\right\| \leq 10^{-5}$.

In the early conjugate gradient algorithms, the restart strategy was usually restarted whenever $k=n$ or $k=n+1$. When $\mathrm{n}$ is very large, another efficient restart is called Wolfe restart, which is defined by :

$\left|g_{k+1}^{T} g_{k}\right| \geq 0.2\left\|g_{k+1}\right\|^{2} \quad$ [9], using this strategy, the new algorithm in the table below performs better than the standard algorithm for most of the problems, depending on the 
Number of iteration(NOI), and depending on the Number of functions (NOf) with different dimensions $(\mathrm{N})$.

The Numerical Results for the new Algorithm

\begin{tabular}{|c|c|c|c|c|c|c|}
\hline \multirow[t]{2}{*}{ NO } & \multirow[t]{2}{*}{ Test Fun. } & \multirow[t]{2}{*}{ Dim. } & \multicolumn{2}{|c|}{$\begin{array}{c}\text { Standard } \\
\text { Histen\Steven } \\
\text { algorithm }\end{array}$} & \multicolumn{2}{|c|}{$\begin{array}{l}\text { Modified } \\
\text { algorithm }\end{array}$} \\
\hline & & & NOI & $\mathrm{NOF}$ & NOI & NOF \\
\hline 1 & Arwhead (Cute) & 1000 & 17 & 122 & 13 & 66 \\
\hline 2 & Broyden Tridiagonal & 1000 & 33 & 58 & 35 & 60 \\
\hline 3 & Diagonal 2 & 1000 & 177 & 300 & 263 & 439 \\
\hline 4 & DIXMAAN A (Cute) & 1000 & 6 & 12 & 6 & 12 \\
\hline 5 & DIXMAAN E (Cute) & 1000 & 259 & 413 & 210 & 324 \\
\hline 6 & DIXMAAN F (Cute) & 1000 & 232 & 447 & 204 & 366 \\
\hline 7 & DIXMAAN G (Cute) & 1000 & 228 & 371 & 213 & 348 \\
\hline 8 & DIXMAAN H (Cute) & 1000 & 244 & 445 & 224 & 387 \\
\hline 9 & DIXMAAN J (Cute) & 1000 & 227 & 444 & 262 & 458 \\
\hline 10 & EDENSCH (Cute) & 1000 & 39 & 353 & 47 & 515 \\
\hline 11 & Extended Cliff & 1000 & 18 & 19 & 18 & 19 \\
\hline 12 & Extended Hiebert & 1000 & 79 & 171 & 78 & 188 \\
\hline 13 & Extended Himmelblou & 1000 & 10 & 19 & 17 & 28 \\
\hline 14 & Extended Powel BD1 & 1000 & 52 & 83 & 14 & 27 \\
\hline 15 & Extended psc1 & 1000 & 6 & 13 & 7 & 15 \\
\hline 16 & Extended Three Expo Terms & 1000 & 12 & 19 & 11 & 19 \\
\hline 17 & Extended Tridiagonal 1 & 1000 & 34 & 53 & 41 & 62 \\
\hline 18 & Generalized psc1 & 1000 & 1141 & 1362 & 457 & 793 \\
\hline 19 & Generalized Tridiagonal 2 & 1000 & 64 & 98 & 49 & 74 \\
\hline 20 & NONDia (Cute) & 1000 & 12 & 24 & 13 & 24 \\
\hline 21 & Penalty & 1000 & 2 & 5 & 2 & 5 \\
\hline 22 & Raydan2 & 1000 & 4 & 9 & 4 & 9 \\
\hline 23 & Rosen Brock & 1000 & 34 & 75 & 35 & 69 \\
\hline 24 & Tridiagonal 4 & 1000 & 4 & 8 & 10 & 20 \\
\hline 25 & Tridiagonal 5 & 1000 & 4 & 9 & 4 & 9 \\
\hline 26 & Trigonometric & 1000 & 29 & 52 & 27 & 48 \\
\hline 27 & White \& Holst & 1000 & 26 & 53 & 32 & 64 \\
\hline & Total & & 2993 & 5037 & 2296 & 4448 \\
\hline
\end{tabular}




\begin{tabular}{|c|c|c|c|c|c|c|}
\hline \multirow[t]{2}{*}{ NO } & \multirow[t]{2}{*}{ Test Fun. } & \multirow[t]{2}{*}{ Dim. } & \multicolumn{2}{|c|}{$\begin{array}{c}\text { Standard } \\
\text { HistenlSteven } \\
\text { algorithm }\end{array}$} & \multicolumn{2}{|c|}{ Modified algorithm } \\
\hline & & & NOI & $\mathrm{NOF}$ & NOI & $\mathrm{NOF}$ \\
\hline 1 & Arwhead (Cute) & 5000 & 10 & 21 & 8 & 18 \\
\hline 2 & Broyden Tridiagonal & 5000 & 77 & 118 & 78 & 121 \\
\hline 3 & Diagonal 2 & 5000 & 509 & 826 & 458 & 768 \\
\hline 4 & DIXMAAN A (Cute) & 5000 & 6 & 12 & 6 & 12 \\
\hline 5 & DIXMAAN E (Cute) & 5000 & 484 & 763 & 562 & 887 \\
\hline 6 & DIXMAAN F (Cute) & 5000 & 534 & 1055 & 545 & 1074 \\
\hline 7 & DIXMAAN G (Cute) & 5000 & 703 & 1191 & 601 & 1022 \\
\hline 8 & DIXMAAN H (Cute) & 5000 & 479 & 793 & 645 & 1015 \\
\hline 9 & DIXMAAN J (Cute) & 5000 & 593 & 1211 & 497 & 987 \\
\hline 10 & EDENSCH (Cute) & 5000 & 91 & 2010 & 62 & 1200 \\
\hline 11 & Extended Cliff & 5000 & 10 & 12 & 10 & 12 \\
\hline 12 & Extended Hiebert & 5000 & 77 & 163 & 81 & 190 \\
\hline 13 & Extended Himmelblou & 5000 & 19 & 31 & 17 & 28 \\
\hline 14 & Extended Powel BD1 & 5000 & 55 & 87 & 55 & 87 \\
\hline 15 & Extended psc1 & 5000 & 7 & 15 & 8 & 17 \\
\hline 16 & Extended Three Expo & 5000 & 14 & 22 & 9 & 16 \\
\hline 17 & Extended Tridiagonal 1 & 5000 & 80 & 1642 & 60 & 957 \\
\hline 18 & Generalized psc1 & 5000 & 230 & 560 & 671 & 992 \\
\hline 19 & Generalized Tridiagonal & 5000 & 60 & 98 & 63 & 95 \\
\hline 20 & NONDia (Cute) & 5000 & 7 & 14 & 8 & 15 \\
\hline 21 & Penalty & 5000 & 2 & 5 & 2 & 5 \\
\hline 22 & Raydan2 & 5000 & 4 & 9 & 4 & 9 \\
\hline 23 & Rosen Brock & 5000 & 35 & 82 & 31 & 62 \\
\hline 24 & Tridiagonal 4 & 5000 & 4 & 8 & 10 & 20 \\
\hline 25 & Tridiagonal 5 & 5000 & 4 & 9 & 4 & 9 \\
\hline 26 & Trigonometric & 5000 & 19 & 39 & 19 & 39 \\
\hline \multirow[t]{2}{*}{27} & White \& Holst & 5000 & 26 & 53 & 42 & 85 \\
\hline & Total & & 4139 & 10849 & 4556 & 9742 \\
\hline
\end{tabular}




\begin{tabular}{|c|c|c|c|c|c|c|}
\hline \multirow[t]{2}{*}{ NO } & \multirow[t]{2}{*}{ Test Fun. } & \multirow[t]{2}{*}{ Dim. } & \multicolumn{2}{|c|}{$\begin{array}{c}\text { Standard } \\
\text { Histen\Steven } \\
\text { algorithm }\end{array}$} & \multicolumn{2}{|c|}{$\begin{array}{l}\text { Modified } \\
\text { algorithm }\end{array}$} \\
\hline & & & NOI & $\mathrm{NOF}$ & NOI & $\mathrm{NOF}$ \\
\hline 1 & Arwhead (Cute) & 10000 & 4 & 10 & 21 & 264 \\
\hline 2 & Broyden Tridiagonal & 10000 & 77 & 123 & 87 & 134 \\
\hline 3 & Diagonal 2 & 10000 & 692 & 1123 & 704 & 1164 \\
\hline 4 & DIXMAAN A (Cute) & 10000 & 6 & 12 & 6 & 12 \\
\hline 5 & DIXMAAN E (Cute) & 10000 & 716 & 1122 & 614 & 953 \\
\hline 6 & DIXMAAN F (Cute) & 10000 & 1095 & 2008 & 829 & 1334 \\
\hline 7 & DIXMAAN G (Cute) & 10000 & 948 & 1591 & 858 & 1499 \\
\hline 8 & DIXMAAN H (Cute) & 10000 & 1438 & 2693 & 917 & 740 \\
\hline 9 & DIXMAAN J (Cute) & 10000 & 1072 & 2147 & 958 & 1573 \\
\hline 10 & EDENSCH (Cute) & 10000 & 53 & 866 & 79 & 1779 \\
\hline 11 & Extended Cliff & 10000 & 11 & 13 & 11 & 13 \\
\hline 12 & Extended Hiebert & 10000 & 77 & 165 & 78 & 186 \\
\hline 13 & Extended Himmelblou & 10000 & 19 & 31 & 17 & 28 \\
\hline 14 & Extended Powel BD1 & 10000 & 35 & 59 & 39 & 67 \\
\hline 15 & Extended psc1 & 10000 & 7 & 15 & 8 & 19 \\
\hline 16 & Extended Three Expo Terms & 10000 & 16 & 26 & 10 & 18 \\
\hline 17 & Extended Tridiagonal 1 & 10000 & 36 & 74 & 108 & 2500 \\
\hline 18 & Generalized psc1 & 10000 & 562 & 1105 & 637 & 2010 \\
\hline 19 & Generalized Tridiagonal 2 & 10000 & 54 & 92 & 59 & 93 \\
\hline 20 & NONDia (Cute) & 10000 & 4 & 7 & 4 & 7 \\
\hline 21 & Penalty & 10000 & 2 & 5 & 2 & 5 \\
\hline 22 & Raydan2 & 10000 & 4 & 9 & 4 & 9 \\
\hline 23 & Rosen Brock & 10000 & 35 & 83 & 31 & 62 \\
\hline 24 & Tridiagonal 4 & 10000 & 4 & 8 & 10 & 20 \\
\hline 25 & Tridiagonal 5 & 10000 & 4 & 9 & 4 & 9 \\
\hline 26 & Trigonometric & 10000 & 17 & 34 & 16 & 32 \\
\hline \multirow[t]{2}{*}{27} & White \& Holst & 10000 & 34 & 74 & 42 & 85 \\
\hline & Total & & 2270 & 13504 & 6153 & 14615 \\
\hline
\end{tabular}


The performance's percentage of the modified algorithm depending on the standard $\mathrm{H} \backslash \mathrm{S}$ algorithm can be summarized in the table below:

\begin{tabular}{|c|c|c|c|c|c|c|}
\hline & \multicolumn{2}{|c|}{$\begin{array}{c}\text { The total of NOI and } \\
\text { NOF for the Standard } \\
\text { HIS algorithm }\end{array}$} & \multicolumn{2}{c|}{$\begin{array}{c}\text { The total of NOI and } \\
\text { NOF for the modified } \\
\text { algorithm }\end{array}$} & \multicolumn{2}{|c|}{$\begin{array}{c}\text { The percentage of } \\
\text { equations that get better }\end{array}$} \\
\hline DIM & NOI & NOF & NOI & NOF & NOI & NOF \\
\hline 1000 & 2993 & 5037 & 2296 & 4448 & $56 \%$ & $63 \%$ \\
\hline 5000 & 4139 & 10849 & 4556 & 9742 & $59 \%$ & $63 \%$ \\
\hline 10000 & 7022 & 13504 & 6153 & 14615 & $56 \%$ & $56 \%$ \\
\hline
\end{tabular}




\section{REFERENCES}

[1] Andrie N. (2008), "An Unconstraint Optimization test functions collection", Adv. Model. Optimization. Vol(10), pp:147-161.

[2] Dai,Y. And Yuan, Y. (1999), "A Nonlinear Conjugate Gradient Method With A Strong Global Convergence Property",Siam J. Optim., Vol(10), pp:177- 182.

[3] Fletcher, R., And Reeves, C., (1964), "Function Minimization By Conjugate Gradients", Comput. J. Vol(7) ,149-154.

[4] Fletcher, R.: Practical Methods of Optimization, vol. I: Unconstrained Optimization. Wiley, New York (1987).

[5] Hestenes, M.R And Stiefel, E. , (1952),"Method Of Conjugate Gradient For Solving Linear Equations", Journal Of Research Of The National Bureau Of Standards , Vol.(5),No.(49), 409-436.

[6] Liu, Y. And Storye, C., (1991) ," Efficient Generalized Conjugate Gradient Algorithms",Part1:Theory,J.Optimization Theory. Appl. Vol.(69),pp:129-137.

[7] M. Mamat and I. Mohd, (2013), "A New Scaled Hybrid Modified BFGS Algorithms for Unconstrained Optimization", Vol.7,No. 6,263-270.

[8] Nicolai Andrei,(2010), "New accelerated conjugate gradient algorithms as modification of Die-Yuan's computational scheme for unconstrained optimization", journal of computational and applied mathematics 234,33973410.

[9] Powel, M.J. D., (1976a),"Restart Procedure For The Conjugate Gradient Method, Mathematical programing", Vol.(1),pp.241-254. 